\title{
INCIDENCIA DEL EMPRENDIMIENTO SOCIAL FEMENINO EN EL DESARROLLO DEL CUSCO, UNA MIRADA DESDE LA EDUCACIÓN
}

\section{INCIDENCE OF FEMALE SOCIAL ENTREPRENEURSHIP IN THE DEVELOPMENT OF CUSCO, A LOOK FROM EDUCATION}

\author{
Mabel Iñakapalla Chávez Bermúdez ${ }^{(1)}$ \\ Universidad Nacional de San Antonio Abad del Cusco, Perú
}

\begin{abstract}
Resumen: En el año 2021 el Perú cumplirá su segundo centenario de historia republicana. A lo largo de este periodo, se ha presentado una gran dicotomía entre la economía y el desarrollo social de los sectores más vulnerables, quienes aún carecen de los principales servicios básicos, acceso a la educación, la alimentación, la salud y a la cultura. Esto es un gran problema social que el Estado no puede abarcar. Frente a ello, en las dos últimas décadas han surgido distintas iniciativas de emprendimientos sociales femeninos que han incidido de manera directa en la solución de los problemas y necesidades de nuestra sociedad. Existe una preponderancia femenina de identificación con la realidad social generando así una nueva forma de participación social, económica y política que se involucra directamente con la población y sus necesidades. En el presente artículo se ha considerado la experiencia de la Asociación Civil Pukllasunchis y la Asociación Educativa Aprender, lideradas por mujeres, quienes han propuesto alternativas educativas que promueven el desarrollo social en Cusco, bajo un enfoque humanista, fundamentado en esta visión: "no se puede ayudar a los demás si no te ayudas a ti mismo".
\end{abstract}

Palabras clave: emprendimiento femenino, emprendimiento social, desarrollo social, educación y desarrollo, participación social femenina.

Abstract: Throughout Peru's two-hundred years as an independent republic there has always been a dichotomy between economy and society, where economic and market interests have prevailed over social development goals: education, healthcare, culture environment. However, in this new century social entrepreneurships can now have a direct influence on providing solutions to social needs, and they can also promote socioeconomic developments in the

(1) Magister en Antropología Social- Universidad Nacional de San Antonio Abad del Cusco. Correo electrónico: ichavez_cuscospsa@yahoo.es 
areas where they work. During the past ten years, some of the most impactful social-responsible companies and organizations in the province of Cusco have been promoted by women. In Peru, and similarly like in other countries in Latin America, there is a lack of a proper legal framework for social-thriven companies and initiatives, making them a bit overlooked. To analyze these companies, we required an interdisciplinary approach and proposed an appropriate definition for this type of companies that look to have a social impact. For this research work, the following women-led companies from the Region of Cusco were considered: Asociación Civil Pukllasunchis and the Asociación Educativa Aprender.

Key words: feminine entrepreneurship, social entrepreneurship, social development, education and development, feminine social participation. 


\section{La participación de la mujer en el desarrollo social de Cusco a través del emprendimiento social}

Es importante conocer los diferentes tipos de emprendimiento social, estos siempre han sido considerados desde el género masculino. Existe así, un vacío en torno al aporte de la mujer al desarrollo social. En este siglo, la mujer ha tomado un rol protagónico, algo que se le había negado por mucho tiempo: ser parte activa de la sociedad. Se tomará en consideración un año referencial: $1956^{(2)}$. En el Perú, las mujeres obtuvieron el derecho de participar en las elecciones generales solamente hace 62 años, de los 135 años de su condición de República que tenía el país en ese entonces. Por esta razón se hace necesario el estudio de los tipos de participación social, política y económica de las mujeres en la sociedad peruana contemporánea.

El emprendimiento es un término que ha sido empleado con mayor frecuencia en Perú en las dos últimas décadas (1990- 2000), siendo considerado como uno de los países más emprendedores del Mundo, según el reporte anual de The Global Entrepreneurship Monitor (GEM) de los cinco últimos años (2012- 2017). El emprendimiento se considera como un fenómeno social, económico y cultural que busca el cambio social desde una perspectiva diferente: el compromiso y la identificación plena con los grupos sociales que vienen siendo menos favorecidos.

La presente investigación aborda el tema del emprendimiento social femenino en la ciudad del Cusco como una actividad empresarial para atender las necesidades de grupos sociales menos favorecidos o vulnerables, que son desatendidos por el Estado. Estos proyectos son liderados por mujeres que se ven identificadas con la problemática social de una manera distinta a la de los hombres, concatenando sus vidas personales hacia el desarrollo de sus emprendimientos, basados en la empatía y la relación directa con los usuarios de los servicios. De esta manera, el emprendimiento social femenino se puede considerar un fenómeno que genera cambios sociales sustanciales en el contexto en el que se desenvuelven, promoviendo el desarrollo sostenible en el sector social de la comunidad donde realizan sus acciones.

En este artículo se toman dos casos de estudio: la ONG Aprender, dedicada al servicio educativo de niños, niñas y jóvenes con algún tipo de discapacidad o discapacidades (física, mental o psicológica), enmarcado dentro de la Educación Básica Especial y la ONG Pukllasunchis que mediante su Institución Educativa abarca el servicio de la Educación Básica Regular, con énfasis en la interculturalidad, la equidad de género y la inclusión de niños y niñas con discapacidad.

(2) La participación de las mujeres en las elecciones peruanas, fue oficializada en el Gobierno de turno de Manuel Arturo Odría, Presidente Constitucional de la República del Perú (28 de julio de 1950. 28 de Julio de 1956). 


\section{La educación como uno de los principales problemas de Perú}

La educación en el Perú fue declarada en emergencia en el año $2003^{(3)}$ generándose a partir del Ministerio de Educación, un Programa Nacional de Emergencia Educativa (PNEE) ejecutado en el periodo de 2004 al 2006, mediante el cual se planteaban una serie de acciones cuyo objetivo fue mejorar el sistema educativo a través de diversas instancias burocráticas, que a lo largo del proceso tuvieron muchas dificultades para llegar directamente a mejorar las escuelas a nivel nacional ${ }^{(4)}$. Actualmente, el sistema educativo peruano aún se encuentra en un proceso de implementación pedagógica y ampliación limitada en el Presupuesto General de República (PGR).

Por otro lado, de acuerdo al informe económico del Fondo Monetario Internacional (FMI) para el año 2017 en el Perú se tuvo previsto un crecimiento del $\mathrm{PBI}$ de $4.3 \%$, lo que significa una mayor inversión en el gasto público nacional ${ }^{(5)}$. Estas cifras económicas alentadoras significan la reducción de la pobreza, pero tan sólo en cifras porcentuales, ya que la desigualdad social, sigue siendo un gran problema a nivel nacional.

Este crecimiento económico debería ser compatible con la formulación de políticas de Estado, que se enfoquen a la igualdad de oportunidades a nivel social, incidiendo en el acceso a la educación y la salud, con tres ejes transversales: a) la igualdad de género, b) la cultura y c) la protección del medio ambiente.

El crecimiento económico de la provincia de Cusco durante el periodo de 2001-2014 ha tenido un gran crecimiento, evidenciado en el Producto Bruto Interno (PBI) que ha alcanzado el 91\%, cifra porcentual que internacionalmente supera a China y Panamá. Esta evolución de la economía se debe fundamentalmente a la minería y a los proyectos de energía, sectores que para el año 2014, representaban el $42 \%$ del PBI regional ${ }^{(6)}$.

El presupuesto asignado a la educación peruana es uno de los más bajos a nivel latinoamericano. Los medios de comunicación refieren: "El Perú es uno de los países de América Latina que menos porcentaje del Producto Interno Bruto (PBI) destina al sector educación. Así lo señaló un reciente estudio del Banco Interamericano de Desarrollo (BID). Nuestro país invierte solo un 3,7\%." (7)

(3) Programa Nacional de Emergencia Educativa (PNEE) Julio de 2003. Ministerio de Educación del Perú. Recuperado de http://spij.minjus.gob.pe/Normas/textos/200803T.pdf. Fecha: 20/ 09/2017

(4) Montero, Gonzáles, Eguren, Uccelli, De Belaunde. “El Estado de la Educación” Estudios sobre políticas, programas y burocracias del sector. Instituto de Estudios Peruanos (IEP). Agosto 2009

(5) Recuperado de http://peru21.pe/economia/peru-liderara-crecimiento-economico -20172268677. Fecha 20/09/2017.

(6) III Foro de Desarrollo Económico Social, organizado por el Instituto Peruano de Economía (IPE) y la Universidad Nacional de San Antonio Abad del Cusco (UNSAAC). Recuperado de: http://elmontonero. pe/economia/el-boom-economico-del-cusco. Fecha: 12 de enero de 2017.

(7) Acuña Esteban. ¿Sabes cuánto invierte el Perú en educación? EI BID nos brinda algunas cifras. 
Con el objetivo de profundizar el contexto de la investigación de los emprendimientos sociales femeninos, vinculados a los servicios educativos, se hará un análisis del contexto de la discapacidad y Educación Básica Especial (EBE), la Educación Básica Regular (EBR) a nivel local y nacional.

\subsection{La Educación Básica Especial en el Perú: Un largo camino hacia la verdadera inclusión.}

De acuerdo a la Dirección General de Educación Básica Especial (DIGEBE) (8) durante los últimos 40 años se han considerado diversas políticas en el sistema educativo peruano. Estas han oscilado desde las propuestas de las Instituciones Educativas especiales separadas de la Educación Básica Formal, hasta el actual modelo educativo, basado en un enfoque de derecho a la educación de calidad e igualdad de condiciones y oportunidades, tal como se propuso la Convención sobre los derechos de las personas con discapacidad en su Art. 24 (ONU- 2006) ${ }^{(\mathbf{9})}$. En 1971 se creó un órgano normativo que ubicaba a la Educación Especial como una modalidad del sistema educativo, responsable de la formulación de políticas y las orientaciones pedagógicas para el desarrollo de la educación especial en Perú. Posteriormente, en la década de los 80 se establecieron Centros de Educación Especial (CEE) en los niveles de educación inicial y primaria. También se promovió la formación laboral de los jóvenes que presentaban algún tipo de retardo. Cabe indicar, que en los espacios geográficos donde no había un CEE se abrieron aulas de educación especial en colegios regulares, bajo un sistema paralelo y segregado.

En los 90 se elaboró el Proyecto de Integración de Niños con Necesidades Especiales a la Escuela Regular, que fue asesorado por la UNESCO, a través del cual se incorporaron por primera vez los estudiantes con discapacidad a los colegios regulares. En el año 2003 se promulgó la nueva Ley General de Educación, № 28044, en la que se aborda el derecho a la educación de los estudiantes con discapacidad bajo una concepción de educación inclusiva. En 2006 se crea la Dirección Nacional de Educación Básica Especial (D.S. Nº06-2006-ED), de esta forma logra autonomía de la educación inicial y primaria.

La Educación Básica Especial (EBE), constituye un gran reto para el Sistema Educativo peruano, por ello se hacen necesarias las investigaciones en torno a los procesos pedagógicos, sociales, psicológicos y antropológicos tanto de los principales usuarios, así como de las familias y la sociedad en conjunto.

Diario Perú21. Recuperado de: https://peru21.pe/peru/invierte-peru-educacion-373881. Fecha 24 de marzo de 2018.

(8) Dirección General de Educación Básica Especial. Educación Básica Especial y Educación Inclusiva Balance y perspectivas. Año 2012. Recuperado de http://www.minedu.gob.pe/minedu/ archivos/a/002/05-bibliografia-para-ebe/9-educacion-basica-especial-y-educacion-inclusiva-balance-y-perspectivas.pdf

(9) CONVENCIÓN SOBRE LOS DERECHOS DE LAS PERSONAS CON DISCAPACIDAD Recuperado de: http://www.un.org/esa/socdev/enable/documents/tccconvs.pdf 


\subsection{Enfoques de la Educación Peruana Básica Regular}

En los últimos treinta años a nivel nacional se ha discutido acerca del sistema educativo peruano, pese a que en diversos periodos presidenciales han sido innovadas y reformuladas las políticas educativas, aún no se han generado ni evidenciado logros coherentes a la realidad nacional. El sistema educativo republicano en Perú ha pasado por un proceso histórico basado en la aplicación de modelos pedagógicos importados, como el francés, inglés, norteamericano que devienen en la actual realidad educativa peruana. Es así, como estas experiencias educativas no tomaron en cuenta el contexto cultural, social e histórico de un país tan diverso como el nuestro.

Por ello, una primera inferencia que deriva de este antecedente es vincular directamente la educación y la cultura como dos factores preponderantes para el proceso educativo nacional, que muy aparte de nominaciones como las de "Educación Intercultural Bilingüe" puedan reflejar lo que verdaderamente somos, es decir la identidad de cada población o grupo étnico. A nivel nacional existen diversas iniciativas en cuanto a políticas educativas y culturales por parte del Estado, que en la mayoría de los casos no logra una interacción entre los diversos sectores ministeriales. El trabajo interinstitucional es importante para generar políticas y proyectos que promuevan acceso al derecho a la educación. Estos temas deben ser tratados de manera transversal desde la política de gobierno: la educación y la cultura vinculadas entre sí para promover desarrollo integral.

Uno de los principales objetivos del Proyecto Educativo Nacional (PEN) $)^{(\mathbf{1 0})}$ para el siglo XXI es que la educación pueda contribuir a la formación de todas las personas sin exclusión, con derecho a tener una educación de calidad, así como los ciudadanos conscientes de sus derechos y sus responsabilidades, con una ética sólida, procurando el bienestar de sí mismos y de su entorno social y medio ambiental, para que de esta manera se logre una edificación colectiva de la democracia y del desarrollo del país.

El Perú es un país multicultural y plurilingüe, por ello un modelo educativo basado en la identidad cultural de cada territorio es el punto de partida para lograr resultados visibles en este contexto educativo nacional y que se vea reflejado en la población peruana.

Una experiencia educativa en los primeros años de desarrollo influye en el desenvolvimiento social de la persona a lo largo de su vida, por ello es importante considerar que la educación debe comprender ciertos temas de acuerdo a su realidad social. El sistema educativo peruano debe adecuarse al entorno donde se desenvuelven los actores del aprendizaje. La identidad se ha de formar y fortalecer

(10) Proyecto Educativo Nacional: Una respuesta integral, el Proyecto Educativo Nacional. Lima noviembre de 2006. Pág. 39 
en base a las experiencias y conocimientos que se den en estos primeros años de aprendizaje. Por otro lado, la educación se adecúa a cada estudiante, debido a que cada ser humano es distinto incluso de sus coetáneos.

La diversidad cultural del Perú es una de las simientes más importantes que puede constituir un factor de desarrollo a futuro, por ello se hace necesaria la interrelación entre los diversos estamentos ministeriales a nivel nacional, así como de los gobiernos locales, comenzando desde los espacios más significativos como el hogar y la escuela con la participación de los docentes y de los padres y madres de familia. Tenemos un país tan diverso que el recurso cultural debería ser el impulso y el eje central de cualquier política sobre todo educativa.

\section{Aprender: una forma especial de educar a través del amor}

La Asociación Civil sin fines de lucro "Aprender" es liderada por Maritza Mayorga Molina, cusqueña de 42 años, quien estudió en el Instituto de Educación Superior Pedagógico Santa Rosa de Cusco. Luego de su paso por los principales Centros de Educación Especial (CEBEs) de la ciudad, en el año de 2010 inicia su propio emprendimiento social educativo, bajo la forma societaria de Asociación Civil sin Fines de Lucro ${ }^{(11)}$, conformada también por su hermano, Abel Valdez Molina y su madre Rosa Abigail Molina Figueroa. Una de las principales características de los emprendimientos sociales es la participación familiar directa, lo cual destaca también en Aprender, debido a que el capital más importante es el humano.

Maritza Mayorga, atiende anualmente en promedio 14 niños, niñas y jóvenes que presentan algún tipo de discapacidad (física o mental: síndrome de Down, autismo, hiperactividad, parálisis y niños con plurideficiencia) que oscilan entre los 3 a 18 años $^{(\mathbf{1 2 )}}$ que son procedentes de las zonas urbana y rural del Cusco. El objetivo de esta asociación, de acuerdo a la referencia de su fundadora es apoyar a los niños con necesidades especiales y hacer un acompañamiento directo y constante a sus familias. (Entrevista Maritza Mayorga (42 años- 13 de marzo 2017).

El local de Aprender se encuentra ubicado en la calle Quiscapata, en el Distrito de Santiago - Cusco, a inmediaciones de la Plaza de Armas del mencionado distrito. Los días sábados se convierte en una calle transitada por los vendedores y compradores del Baratillo ${ }^{(13)}$, quienes no advierten la existencia de esta Asociación. Las instalaciones de Aprender son compartidas con la vivienda familiar de Maritza, esto le da la facilidad de manejar y organizar sus tiempos de acuerdo a los requerimientos que demanda este servicio educativo a los distintos casos de sus niños y niñas, así como la interacción con los familiares.

(11) Acta de Constitución y Aprobación de Estatutos de la Asociación Civil Sin Fines de Lucro "Aprender". Ver Anexo 2.

(12) Las visitas a la Asociación Civil sin Fines de Lucro Aprender se realizaron durante el mes de marzo del año 2017, durante el horario de atención regular 8:00 am a 2:00 pm.

(13) Feria sabatina de venta e intercambio de objetos de segundo uso. 
La primera vez que visité Aprender, me sorprendió el grado de concentración de los niños frente a su bastidor de tejido. Alrededor de 10 niños y niñas entre 3 a 18 años enfocan toda su atención al avance del tejido y a las instrucciones de su maestra, que, con inagotable tolerancia y dedicación, pero a la vez exigencia, enseña el avance del tejido. Maritza les hace desatar una y otra vez a quienes se equivocan, una de las niñas le refuta, pero sigue con su tarea. Las dos primeras horas de mi participación en la sesión de clases, percibo que los niños y niñas omiten mi presencia, pero me observan con duda, de vez en cuando, a manera de sigilosos evaluadores de mi función en ese espacio. Lo que me sorprende más, durante esa primera sesión es la habilidad psicomotriz que los participantes han desarrollado, comparada con algunos grupos de niños y niñas que tuve la oportunidad de conocer en colegios de educación básica regular.

Esa primera mañana logré aprender mucho de aquellos niños y niñas y sus bastidores en los que no sólo tejían lanas de colores sino destejían mis prejuicios sobre los niños llamados con "habilidades diferentes". Llegando a la conclusión que aquella diferencia era una distinción que más los aproxima a la tolerancia y al aprendizaje significativo de vivir en una sociedad tan llena de prejuicios y necesidades sociales.

Al terminar la sesión de clases de aquel primer día, los niños y niñas de "Aprender" se despiden muy cariñosos como si hubiésemos sido compañeros de varias sesiones de inter aprendizaje. Las madres llegan presurosas de sus centros laborales o sus hogares para recoger a sus hijos e hijas, la mayoría de ellas preocupadas por haberlos hecho esperar demasiado. Maritza las recibe con mucha amabilidad y les indica a sus alumnos y alumnas que no deben olvidar sus pertenencias. Los despide con un abrazo que da la mejor imagen de agradecimiento tanto del alumno como de la maestra. Maritza está extenuada pero no pierde la sonrisa con ellos, durante la sesión de aprendizaje no sólo es profesora, sino también auxiliar, se encarga de atender todas sus necesidades fisiológicas y también emocionales. No es un trabajo fácil, muchos de ellos vienen de mal carácter, rebeldes o ansiosos. Pero ¿qué motiva a esta mujer a emprender semejante tarea?

Durante la entrevista refiere que la mayor motivación para iniciar y continuar con el proyecto fue espiritual:

Es algo más espiritual, yo trabajaba en un centro especial estatal y cierto día que me pongo mal, al despertar, al día siguiente, lo primero que se me vino a la cabeza era que estaba desperdiciando mi vida y que había muchas cosas que podía hacer yo, de acuerdo a mi criterio, porque no comulgaba con algunas formas, en este caso puede ser del sistema mismo, entonces yo sentía que había... y se le podía dar un rumbo distinto a todo eso ¿no? es más una motivación espiritual... (Entrevista Maritza Mayorga (42 años- 13 de marzo 2017) 
Maritza manifiesta que su mayor logro es el hecho de haber incidido en la felicidad de los niños, niñas, jóvenes y de las familias con las que ha trabajado:

La mayor satisfacción, la cosa más linda que te puede dar todo esto es justo lo que vi ayer, en uno de los chicos: su sonrisa, su felicidad, el hecho de que como ser humano se sienta bien y que tu veas es a felicidad impregnada en su rostro, para mí es la cosa más linda de este mundo, o sea yo con eso me siento bien pagada, de verdad... no importa si es uno o si son diez o veinte no me importa mucho cuantos son pero si me importa que los pocos o muchos que estén, yo sienta, yo vea que son personas que se sienten felices, ese es mi mayor logro, mi mayor meta, mi mayor ansia respecto a cada uno de ellos. (Ibid.)

Maritza Mayorga da énfasis a la interrelación con la familia de los niños y niñas atendidos en Aprender, generándoles mayores espacios en los que se puedan dialogar sobre los problemas y necesidades tanto de los niños y niñas, así como de sus familias. Así mismo, refiere que, al hablar de una inclusión social se debe empezar por la familia:

En un futuro, visualizo a las familias mucho más involucradas, con madres, padres y hermanos y todos los que estén alrededor de los chicos trabajando por ellos, es lo que en este momento aspiro, me encantaría, pienso que los que tienen que estar involucrados de verdad son las familias, porque si no se hace una inclusión familiar en vano podemos hablar de una inclusión social ¿no? porque la inclusión tiene que empezar en casa. (Ibid.)

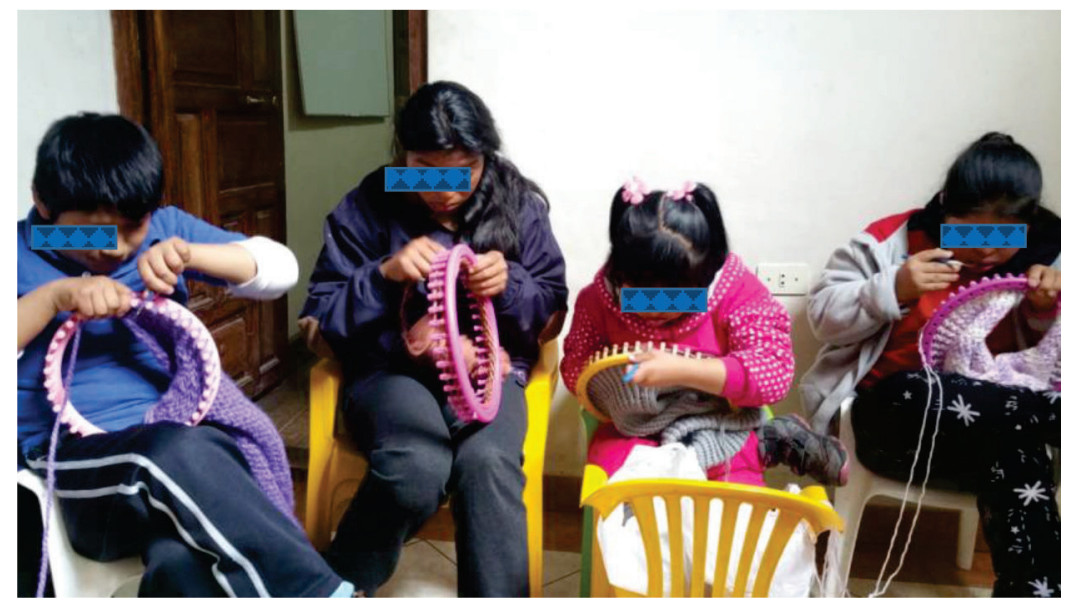

Foto: Niños y niñas elaborando tejidos en su bastidor circular-Aprender. Fecha: 16 de junio de 2018 
Una de las políticas educativas que se promueve dentro del actual sistema nacional de la educación es la inclusión social, Maritza Mayorga, posee una opinión, basada en su experiencia frente al tema:

La inclusión es buena y ni siquiera se tiene que hablar de ello cuando una persona tiene una patología que no tenga que ver con el retardo, es decir, si es un ciego puro puede perfectamente incluirse, porque hacer las adaptaciones no son difíciles ¿no? Es más, todo profesor está capacitado a diversificar el curriculum, y como te digo si no hay compromiso cognitivo, no veo una mayor dificultad en que éstas personas puedan ser incluidas pero cuando tú tienes una dificultad cognitiva eso si es más complejo, y más aún ahora que el sistema te exige que el aula sea un espacio totalmente competitivo, entonces que haces tú poniendo a un niño que tiene retardo... ¿y el retardo que te trae? el retardo, tú sabes que su aprendizaje va a tener un ritmo que va a ser diferente al de los "niños regulares" ¿no? casi siempre va haber olvido, ese olvido te invita a retroceder en lo que has hecho para impulsarte nuevamente, entonces no es fácil y yo si considero que en ese contexto la inclusión para mí es hasta cruel, porque en principio estás perdiendo o le estas quitando el tiempo que nunca más va a volver...(lbid.)

Otro punto muy importante que Maritza refiere en cuanto a la motivación por realizar un emprendimiento social es que no existe una diferenciación definida por el género sino por la espiritualidad de cada ser humano así como su sistema de valores.

No lo sé, a mí no me gusta dividir mucho en que si somos varones o mujeres, me parece absurdo eso, lo que pienso es que a todos se nos ha metido en la cabeza de que el varón es el que tiene que ir a trabajar y ganar plata y dar el sostén a su familia, de repente por ahí que ellos cierran la posibilidad de hacer proyectos de este tipo, puede ser, preferiría verlo de esa forma, no sé si solo las mujeres, porque considero que también hay varones que pueden hacer este tipo de cosas y con mucha vehemencia, con mucha dedicación. (Ibid.)

De acuerdo al testimonio de Maritza Mayorga, su mayor motivación es espiritual, ella considera que se debe proporcionar un enfoque más humano a esta población de niños, niñas y jóvenes con discapacidad. También menciona que tuvo el respaldo de su familia, que significa su mayor apoyo para iniciar y continuar con el proyecto. Así también declara que su mayor logro es el hecho de haber incidido en la felicidad de los niños, niñas, jóvenes y de las familias con las que trabaja. Si bien es cierto, los servicios de acompañamiento que brinda Aprender, no son 
reconocidos "formalmente" dentro del sistema de educación especial, los padres y madres prefieren este acompañamiento. Maritza ha innovado su metodología pedagógica, refiriendo que se trabaja de acuerdo al ritmo de cada niño o niña, dándole a cada uno lo que necesita, porque se debe considerar la individualidad como un factor preponderante en el proceso educativo. El proceso educativo de los niños, niñas y jóvenes de Aprender, de acuerdo a Maritza, se ve influenciado directamente por el estado emocional de los tutores (maestra, madre y padre de familia), por ello debe trabajarse en el desarrollo personal de las personas que acompañan constantemente a los menores. Con respecto a la preponderancia de la participación femenina en los emprendimientos sociales, la fundadora de Aprender menciona que no depende del género sino de los valores y el desarrollo espiritual de cada ser humano, que permite tener una mejor noción del entorno que les rodea. El Estado peruano aún no ha determinado un sistema específico de promoción y fomento de este tipo de emprendimientos sociales, tal como Maritza lo ha referido.

\section{Pukllasunchis: Jugando a Aprender}

La Asociación Civil Pukllasunchis es una Asociación sin fines de lucro dedicada a la educación, con propuestas transformadoras dentro del sistema educativo peruano y en espacios no escolarizados, con un enfoque intercultural, inclusivo y medioambiental ${ }^{(14)}$.

Cristina Appenzeller es considerada como la "madre" fundadora de este proyecto educativo que cumple 30 años en Cusco. La historia de vida de esta mujer está ligada directamente a Pukllasunchis. En el año 1981, junto a un grupo de colaboradores y educadores apasionados por la educación peruana, organiza e implementa las simientes de la Asociación Pukllasunchis:

...Al inicio, eso fue en el 79-1980, yo estaba primero sola, después poco a poco hemos ido haciendo equipo con la gente de acá de la localidad, con cusqueños, y peruanos en general, hemos visto que la educación era muy memorística, muy repetitiva que no formaba en valores, que estaba orientado solamente a lo académico y sobretodo que no formaba en valores y no formaba a personas independientes, autónomas ¿no? y tampoco solidarias, todos esos valores que nosotros formamos en Pukllasunchis que es importante que se trabaje la personalidad de cada alumno, de cada ser humano, pero que también se trabajen los valores colectivos, porque no somos islas, no somos solos, entonces todo ello es muy importante porque la información, los conocimientos en las diferentes áreas o asignaturas eso lo puedes aprender o bajar de google en cualquier momento, entonces viendo

(14) Página web de la Asociación Civil sin fines de Lucro Pukllasunchis. Recuperado de : https://www. pukllasunchis.org 
esa situación, nosotros dijimos, queremos hacer experiencias piloto que puedan contribuir a una educación intercultural, inclusiva, democrática, holística, integral que realmente fomente todas las habilidades de cada chico, sus talentos que pueda descubrirlos, que tenga las condiciones para desarrollarlos. Entrevista a Christine Appenzeller (67 años) - 10 de mayo de 2017

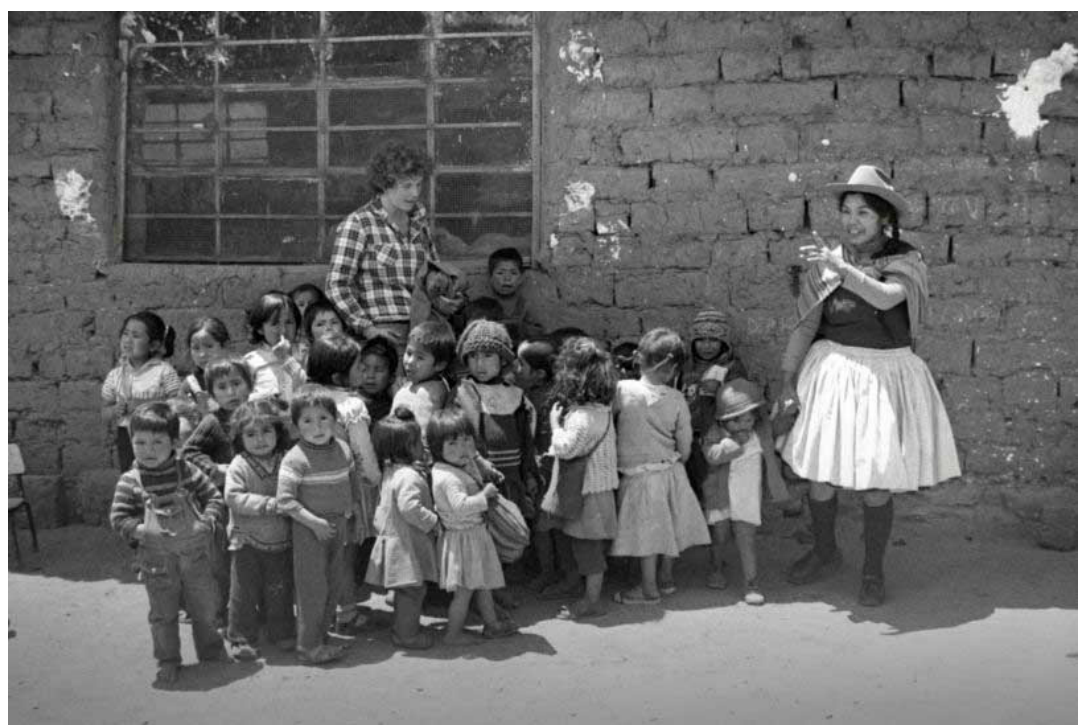

Foto: Cristina Appenzeller en uno de los jardines implementados en la década de los 80 's. Fuente: www.pukllasunchis.com

Una de las principales características de los emprendimientos sociales femeninos es la participación directa de la familia de las iniciadoras de estos proyectos sociales, siendo entonces un recurso humano muy importante para el desarrollo y crecimiento de este tipo de iniciativas. La madre de Cristina Appenzeller ha significado un gran pilar en la historia de Pukllasunchis:

Al principio fue un poco difícil para mí. Sobre todo, para mis papás, pero mi familia siempre me apoyó y en Suiza mi madre con amigos creó una fundación "Pukllasunchis, escuelas para Cusco" y eso existe hasta hoy y recaudan fondos de diferentes personas, organizaciones, mi madre ha trabajado hasta el año 2014, hasta los 84 años ella ha trabajado en todo eso, era la presidenta de allá... (lbid.)

Hasta el año 1987, se realizaron investigaciones sobre diferentes temas educativos de la región, y empezaron a realizar capacitaciones a docentes del nivel 
inicial y primario en metodologías innovadoras pedagógicas. En el año 1988 se crea un centro educativo piloto dirigido a niños y niñas de diversos sectores sociales de Cusco, con el objetivo de desarrollar propuestas educativas que incluyan el enfoque de la interculturalidad, la inclusión social y el cuidado del medio ambiente. El local se ubicaba en el sector de K'ari Grande.

En el periodo de 1990 y 1996, la Asociación Pukllasunchis impulsó diversos programas de capacitación dirigido a docentes en servicio. Esta serie de capacitaciones benefició a un promedio de 80 docentes en promedio anual e indirectamente se beneficiaron alrededor de 3200 estudiantes de instituciones educativas públicas. En 1995, el Ministerio de Educación, reconoce la experiencia en formación pedagógica de Pukllasunchis y les encomienda la capacitación de los docentes estatales de toda la provincia de Cusco, dentro del marco del Plan Nacional de Capacitación Docente (PLANCAD). Así, entre 1995 y 2001, fueron capacitados entre 500 profesores y profesoras al año. Posteriormente, en convenio con la Dirección Regional de Educación Cusco, se ofreció el programa de Actualización Docente, en beneficio de unos 100 docentes de educación inicial y los primeros grados de primaria. En 1998 se crea el programa de Educación Intercultural Bilingüe, para capacitar a docentes de zonas rurales cercanas a la ciudad de Cusco, y con el objetivo de atender a niños y niñas quechua hablantes con un conocimiento incipiente del castellano.

Desde el año 2000 se ejecutaron proyectos específicos a partir de la experimentación en el Centro Educativo Pukllasunchis: Inclusión Escolar y Laboral, Quechua como Segunda Lengua, "Kawsay"- Centro de Promoción Ambiental y Talleres Productivos. Las propuestas validadas son difundidas a través de procesos de capacitación docente en instituciones educativas estatales y privadas de Cusco y otras regiones del Perú.

En el año 2003 se emprende un nuevo proyecto: "Radio con Niñas y Niños Andinos" en el que se producen programas radiales con niños, niñas, padres y madres de familia de zonas alto andinas de Cusco y Puno. A través de este proyecto, se realizan también programas de capacitación y de apoyo pedagógico para docentes de las escuelas participantes. Asimismo, se logra trabajar con organizaciones de base, particularmente con mujeres, quienes también producen programas radiales y otros materiales interculturales para las escuelas.

Desde 2011 inició con el Proyecto de Jóvenes "Sipas Wayna", a través del cual se ofrecen diversas alternativas de desarrollo personal para jóvenes entre 12 y 25 años en espacios de educación no formal. En 2015, se crea el Instituto Superior Pedagógico Pukllasunchis, en el que se ofrece una formación pedagógica intercultural e inclusiva para la región Cusco, en las carreras de inicial y primaria, con especialidad en Educación Intercultural Bilingüe (EIB). 


\section{Burocracia y Políticas Educativas}

La creación o el funcionamiento de un emprendimiento social implica una serie de procesos burocráticos que se dan a partir de las diferentes instancias del Estado Peruano. Debido a que no existe la figura legal que enmarque este tipo de emprendimientos, son signados como Organizaciones No Gubernamentales (ONGs). Cristina refiere que hace 30 años, el proceso burocrático era menor al actual, pero que ahora están fiscalizados por la Agencia Peruana de Cooperación Internacional (APCl) y por otras entidades públicas del Ministerio de Educación, quienes hacen diferencias sin fundamento entre Instituciones Educativas Públicas y Privadas:

... Pero antes de verdad no teníamos trabas y también de parte de la DREC, había mucha apertura, estaban interesados en que nosotros traíamos apoyo para construir colegios o jardines infantiles que pasaban luego al Estado, ahora se ha vuelto un poco más complicado sobre todo muchas exigencias hacia las organizaciones privadas, así como por ejemplo ahora para el colegio, como ha habido esos sucesos de violaciones en algunos colegios privados, y de los públicos ni se habla... (lbid.)

Appenzeller, considera que las políticas educativas a nivel nacional están definidas y bien fundamentadas, pero falta aplicarlas a través de la formación pedagógica de los docentes:

El asunto es que lo que está en el papel y las políticas del Ministerio de Educación y también de la región en principio lo que está en papel está bien. Es justamente inclusivo, intercultural, basado en competencias, en la formación de valores, el problema es que el estado no invierte en formación docente entonces siguen saliendo los docentes con una formación en educación tradicional y no funciona, todo lo que hay en el papel no se aplica. Las políticas en el papel están bien definidas pero no se aplica, por ejemplo como te digo, era revolucionario enseñar quechua como segunda lengua en zonas urbanas ahora está en el currículo y están en la directivas a nivel nacional y regional que hay que enseñar en lengua materna o en zonas vernáculo hablantes hay que enseñar esas lenguas como segunda lengua pero eso está solamente en el papel, no se da recursos, no se forma al personal, si nosotros mismos tenemos problemas en el colegio para encontrar profesores de inicial que también hablen quechua por ejemplo. Ahora estamos formando, porque si tú en 5 años enseñas $a$ un profesor quechua para que pueda enseñar quechua, lo puedes lograr pero hay que invertir, entonces en si en la concepción, en la política no estamos mal, hay cosas muy interesantes pero no se aplica y luego muchas veces, 
ahora por ejemplo todo es "rutas de aprendizaje" pero ahora ya está hablando el ministerio de que dentro de 2 años eso ya no se va a aplicar, el problema es que todo el tiempo cambian las cosas y los profes primero tienen dificultades en aprenderlo y luego cuando ya más o menos lo han aprendido se cambia, no hay continuidad. En cada gobierno viene otra vez algo nuevo(lbid.)

Cristina, considera que, para emprender cualquier proyecto, uno debe conocerse y reconocerse frente a los demás, estableciendo un equilibrio entre sentir, pensar y hacer:

Para cualquier persona y más para los emprendedores o emprendedoras lo más importante es el autoconocimiento y eso es lo que fomentamos también en el colegio, conocerse y saber. Todos somos seres humanos pero cada ser humano tiene diferentes talentos y habilidades entonces para saber qué emprendimiento quiero desarrollar, tengo que conocerme, no voy a seguir lo que hacen todos, porque yo estoy convencida de que en este mundo cada ser humano tiene su lugar pero si quiero comenzar a ocupar el lugar de otro, empiezo a competir por eso en el colegio tampoco fomentamos la competencia, sino que tú mismo te conozcas y puedas decir lo que mejor puedes hacer y eso también va cambiando en el transcurso de la vida pero por eso es muy importante sentir, eso no es cuestión de la cabeza y tiene que ver también un equilibrio entre sentir y pensar, primero sientes y luego usas la mente. Si quieres hacer todo con la mente llegamos a lo que estamos creando con el mundo ahora, destruyendo este mundo, ecológicamente, socialmente, humanamente, que se yo, pero cuando tú estás conectado con tu ser interno, tú tampoco creas algo que hace daño al otro sino creas una empresa social, no vas a hacer plata a costa de otros, no vas a explotar a alguien para ganar tu pero para eso tienes que conectarte contigo mismo tienes que estar no tratando de seguir los paradigmas sociales sino tienes que desarrollar tu talento y tus habilidades y tus potenciales y ahí todo empieza fluir. El logro de estos proyectos es más cualitativo que cuantitativo ¿no? en los emprendimientos sociales. (Ibid.)

Actualmente, el sistema político no puede asegurar un desarrollo social, debido a la corrupción y al manejo que se da en torno a los intereses individuales de cada gobernante, esta afirmación la comparte Appenzeller y refiere:

la política es corrupta y todo... yo si no, me metería, puedo ser emprendedora y puedo contribuir con mi humanidad a crear un mejor sistema educativo pero yo no entraría a un puesto político 
porque me parece un desgaste total y yo prefiero hacerlo desde la vida, desde el campo, no desde la política entonces, pero bueno eso es tanto para el hombre como para la mujer, pero yo no soy parte de un movimiento feminista, es parte de, yo digo no estoy en contra y no voy a luchar contra eso ¿no? pero no participaría activamente en eso, pero para mí el futuro es el equilibrio, la mujer es tan importante como el hombre y el hombre es tan importante como la mujer y también hay expresiones de homosexualidad, todo es natural ¿no? porque en el universo para mí no existe error, todo tiene una razón de ser, lo que pasa es que solo vemos una ventanita chiquita y no vemos el todo... entonces la energía es mucho más potente que una política y cuando cada uno se valora a sí mismo y se reconecta con su esencia que para mí es el alma o el espíritu tú no necesitas Leyes. (Ibid.)

\section{Conclusiones}

1. Los emprendimientos sociales femeninos citados en esta investigación, poseen diversas similitudes: a) la motivación y compromiso personal con la educación de los niños y niñas que albergan en los proyectos educativos b) consideran la importancia de las relaciones interpersonales directas, en las que se reconocen la responsabilidad hacia los otros, haciendo referencia a tres dimensiones: cognitiva, afectiva y práctica (sentir- pensar y hacer). c) el objetivo de éstos es aportar al sistema educativo de la sociedad cusqueña.

2. Pukllasunchis y Aprender, constituyen proyectos de innovación social en Cusco, debido a sus características: novedad, sostenibilidad y la participación activa de la comunidad educativa. Ambas propuestas educativas, han generado incidencia social en las familias con las que vienen trabajando desde sus inicios, como se ha podido corroborar con los testimonios de las madres de los niños y niñas que son parte de los servicios de Pukllasunchis y Aprender.

3. La participación social de la mujer en Cusco no está vinculada a la política directamente, de acuerdo a las entrevistadas, los cambios pueden darse "desde abajo", con este tipo de emprendimientos sociales, que generan cambios sustanciales en una o mil familias. Para ambas emprendedoras sociales no es importante la cantidad sino la calidad del servicio. Un factor muy importante que destacaron es el crecimiento de la corrupción en el sector público, debido a que las actividades son desarrolladas por compromiso, no por vocación.

4. Los emprendimientos sociales constituyen una alternativa para incidir directamente en el desarrollo social a través de las necesidades sociales más importantes, siendo sus servicios más eficientes que los que brinda el Estado debido a que tienen contacto directo y conocimiento del sector de la población con quien se trabaja. El desarrollo social que promueven se enfoca 
en los valores morales y el desarrollo personal del educando, la solidaridad, la interculturalidad, la equidad de género, la inclusión de personas con discapacidad, el respeto y cuidado del medio ambiente y se proyecta a la comunidad, adoptando valores que generan comportamientos armónicos.

\section{Bibliografía}

ACEBEDO AFANADOR, Manuel \& Velasco Abril, Maribel (2017) Emprendimiento Social Femenino: Prolegómenos Conceptuales y Estudio de Casos, en CIEG, revista del Centro de Investigación y Estudios Gerenciales №27. Barquisimeto, CIEG.

AGUIRRE, A. (Edit). (1997). Etnografía: Metodología cualitativa en la investigación sociocultural. México, DF: Alfaomega

ALVARADO, A., La ética del cuidado (2004). Revista Aquichan (Bogotá, Colombia), no 4, Octubre 2004.

ARAGÓN, F. \& Severi, M. (2002). Características personales de los emprendedores.

Diferencias con los ejecutivos de corporaciones. Tesis de maestría no publicada, Universidad del Cema.

BAUER, Susanne; FINNEGAN, Gerry \& HASPELS, Nelien (2011) Género y Emprendimiento. Guía de formación para mujeres empresarias, San José, Organización Internacional del Trabajo

CÁCERES Rojas Reneé \& Ramos Pacheco, Luz (2017). Emprendimiento Laboral y Empoderamiento de Mujeres Artesanas de la Asociación de Tejedoras "Tejidos Huaycán". Tesis para optar el título profesional de Licenciada en Trabajo Social, Huancayo, Universidad Nacional del Centro del Perú.

CURTO GRAU, Marta (2012) Los Emprendedores Sociales: Innovación al Servicio del Cambio Social, en Cuadernos de la Cátedra "la Caixa" de Responsabilidad Social de la Empresa y Gobierno Corporativo, No13, España, IESE Business School

DEES, J.G. (1998). The Meaning of Social Entrepreneurship, Stanford University: Draft Report for the Kauffman Center for Entrepreneurial Leadership.

FORMICHELLA, M. M. (2002). Educación y Pobreza: Una explicación de los círculos viciosos existentes entre ambas. Tesis de Grado, Departamento de Economía, Universidad Nacional del Sur.

GILLIGAN, C. (1982), La moral y la teoría. Psicología del desarrollo femenino.

GILLIGAN, C. (1982) In a Different Voice: Psychological Theory and Women's Development, Cambridge, Harvard University Press. 
GUERRA, M.J. (1999). Mujer, identidad y espacio público, Contrastes. Revista Interdisciplinar de Filosofía, Vol. 4, 1999, pp. 45-64, p. 46.Castiblanco Moreno, Suelen (2013) La Construcción de la Categoría de Emprendimiento Femenino, en revista Facultad de Ciencias Económicas: Investigación y Reflexión, vol. XXI, No 2, (pp. 53-66). Bogotá, Universidad Militar Nueva Granada.

GUZMÁN, A., \& Trujillo, M.A. (2008). Emprendimiento social: Revisión de literatura. Estudios Gerenciales.

KOHLBERG, L. (1981), The Philosophy of Moral Development, San Francisco, Harper \&Row.

MADRIGAL TORRES, Berta; Arechavala Vargas, Ricardo \& Madrigal Torres, Rosalba (2014) El Emprendedor Social su impacto en la Comunidad: la Percepción del Joven Investigador, Panamá. XIV Asamblea General de ALAFEC.

MAX-NEEF, Manfred A. (1993) Desarrollo a Escala Humana. Recuperado de: https:// www.max-neef.cl/descargas/Max Neef- Desarrolloaescalahumana.pdf

MARTÍNEZ, I. y BONILLA, A. (2000), Sistema sexo/género, identidades y construcción de la subjetividad, València, Publicacions de la Universitat de València, (p. 103).

MORVELÍ, Mario. (2015). Antropología de los Sistema económicos. Impresiones Gráficas Meta Color S.R.L. Cusco- Perú

ROA, M.L. (2005). Definición y alcances del emprendimiento social. Disponible en el sitio web: http://www.boliviaemprendedora.org.bo/ public/lst_biblioteca_rbe/ Ist_biblioteca_rbe_7542_archivo.pdf

SAAVEDRA GARCÍA, María Luisa \& Camarena Adame, María Elena (2015) Retos para el Emprendimiento Femenino en América Latina, en revista Criterio Libre, vol 13, No 22, Bogotá, Universidad Libre

THURÉN Britt- Marie. (1993). El Poder Generizado. El desarrollo de la antropología feminista. Instituto de Investigaciones Feministas. Universidad Complutense de Madrid.

SEN Amartya(1998) Hambre, prensa y democracia. Diario Clarín, 18/10/98

SEN, Amartya (1999b) Desarrollo y Libertad. Editorial Planeta.

SEN, Amartya (2000) ¿Qué impacto puede tener la ética? Presentación en la Reunión Internacional sobre "Ética y Desarrollo" del Banco Interamericano de Desarrollo. Documento incluido dentro de la Biblioteca Digital de la Iniciativa Interamericana de Capital Social, Ética y Desarrollo - www.iadb.org/etica. 